\title{
Albuminuria is controlled primarily by proximal tubules
}

Wayne D. Comper

I read with interest a collection of Reviews commissioned by Nature Reviews Nephrology-including the article by Moeller and Tenten (Renal albumin filtration: alternative models to the standard physical barriers. Nat. Rev. Nephrol. 9, 266-277; 2013) $)^{1}$-that together discuss the importance of each of the main components of the glomerular filtration barrier in preventing albuminuria. Nephrology students who read these articles would get the incorrect impression that the glomerulus and/or podocytes are central to development of albuminuria-little evidence has been reported that these components have a direct role in determining albumin handling and albuminuria. For example, no Review in the series addresses the major phenomenological aspects of albuminuria. That is, intact albuminuria can occur without changes in glomerular permeability or plasma albumin levels; ${ }^{2}$ in nephrotic states, intact albuminuria can increase 100 10,000-fold without corresponding changes in glomerular permeability; ${ }^{2}$ and as yet no evidence has been presented that suggests changes in the glomerular components are directly responsible for hypoalbuminaemia.

A watershed issue in understanding albuminuria was the finding that the glomerular sieving coefficient (GSC) of albumin is 0.03 as directly measured by two-photon microscopy ${ }^{3}$ (also predicted from earlier biochemical and physiological studies), ${ }^{2}$ which is a 50 -fold increase on previously published figures. ${ }^{4}$ This finding indicates that the glomerulus normally leaks nephrotic levels of albumin and, if this albumin was not retrieved by proximal tubular cells, the individual would have a nephrotic condition. Furthermore, it also shows that the glomerulus does not have a major role in governing albuminuria. If there are changes in glomerular permeability then it would only require relatively small changes (more than twofold increase in permeability) to have a profound effect in saturating the retrieval mechanism and creating an albuminuric condition. ${ }^{5}$
Is the measured GSC of albumin at 0.03 correct? Physiochemically, it makes sense. The concept of charge selectivity or charge-related albumin restriction that was proposed to cause the previously reported ultralow GSC are flawed. ${ }^{2,6}$ A GSC of 0.03 would be expected based purely on the size selectivity of a $36 \AA$ radius spherical molecule, such as albumin or Ficoll. ${ }^{7}$

The two-photon microscopy method has been associated with technical issues, with some reports of albumin GSC values $<0.0006 .{ }^{8,9}$ These have been resolved by testing various background settings on the microscope. ${ }^{10}$ Another technical issue raised has been 'out-of-focus' fluorescence, ${ }^{11}$ but this has no physical basis in two-photon microscopy. ${ }^{12}$ It was most likely the result of using uncharacterized high molecular weight $(>650,000 \mathrm{kDa})$ preparations, assumed to be unfilterable but with relatively low molecular weight contaminants $(>100,000 \mathrm{kDa})$ that were filtered and yielded an apparent out-offocus reading in the primary urinary space. Equivalent levels of fluorescence can be measured with the genuine filtration of dextran $(150,000 \mathrm{kDa})$, which has a GSC of $0.004^{10}$ as expected from whole-animal fractional clearance studies. ${ }^{7}$ When using two-photon microscopy, molecular transport probes, such as dextrans and Ficolls, of known GSC should, therefore, always be used as standards when measuring albumin GSC. ${ }^{13}$

The capacity of the albumin retrieval pathway is so high ( $200 \mathrm{~g}$ per day $)^{2}$ that any malfunction associated with it will result in hypoalbuminaemia. Another feature observed with albumin processing in the kidney is that $\sim 1-2 \mathrm{~g}$ of albumin is normally excreted in the urine each day in the form of albumin fragments. These fragments are specifically produced by metabolic processes in the kidney and not elsewhere. ${ }^{3,6,14,15}$ In albuminuric states, it seems that the level of albumin degradation decreases, which corresponds with an increase of intact albuminuria as measured by immunoassay, as the immunoassay does not detect albumin fragments. In nephrotic states, this degradation pathway seems to be totally inhibited, which leads to the large increase of intact albuminuria and without the need to alter glomerular permeability. ${ }^{3,6,14}$ Given that the two potential pathways for albumin processing include the high-capacity retrieval pathway and the low-capacity degradation pathway, any change in them might have a paradoxical influence on intact albuminuria. Changes in high-capacity retrieval will affect hypoalbuminaemia, but will only moderately influence intact albuminuria, whereas changes in the degradation pathway might lead to a major change in intact albuminuria with no effect on hypoalbuminaemia.

The glomerulus does seem to have an indirect role on intact albuminuria. Russo et al. ${ }^{15}$ have studied Cd2ap knockout mice, which have a glomerular phenotype that leads to congenital nephrotic syndrome, in which the mice die after $\sim 5$ weeks. Studies in live Cd2ap knockout mice demonstrate that there are essentially no glomerular permeability changes, but a $>100$-fold increase in intact albuminuria, which is primarily due to inhibition of the albumin degradation pathway. ${ }^{15}$ This finding suggests that signalling from the glomerulus to the proximal tubule turns off the degradation pathway. A similar mechanism is likely to be the same for many glomerular/podocyte structural perturbations. ${ }^{15}$

SalAqua Diagnostics Inc., Suite 277, 331 West $57^{\text {th }}$ Street, New York, NY 10019, USA. wcomper@hotmail.com

Competing interests

The author declares no competing interests.

1. Moeller, M. J. \& Tenten, V. Renal albumin filtration: alternative models to the standard physical barriers. Nat. Rev. Nephrol. 9, 266-277 (2013).

2. Comper, W. D., Hilliard, L. M., NikolicPaterson, D. \& Russo, L. M. Diseasedependent mechanisms of albuminuria. Am. J. Physiol. Renal Physiol. 295, F1589-F1600 (2008). 


\section{CORRESPONDENCE}

3. Russo, L. M. et al. The normal kidney filters nephrotic levels of albumin that is retrieved by the proximal tubule cell: retrieval is disrupted in nephrotic states. Kidney Int. 71, 504-513 (2007).

4. Tojo, A. \& Endou, H. Intrarenal handling of proteins in rats using fractional micropuncture technique. Am. J. Physiol. 263, F601-F606 (1992).

5. Koltun, M. \& Comper, W. D. Retention of albumin in the circulation is governed by saturable renal cell-mediated processes. Microcirculation 11, 351-360 (2004).

6. Comper, W. D. The limited role of the glomerular endothelial cell glycocalyx as a barrier to transglomerular albumin transport. Connective Tiss. Res. 55, 2-7 (2014).

7. Asgeirsson, D., Venturoli, D., Fries, E., Rippe, B. \& Rippe, C. Glomerular sieving of three neutral polysaccharides, polyethylene oxide and bikunin in rat: effects of molecular size and conformation. Acta Physiol. 191, 237-246 (2007).

8. Nakano, D. et al. Multiphoton imaging of the glomerular permeability of angiotensinogen. J. Am. Soc. Nephrol. 23, 1847-1856 (2012).

9. Salmon, A. H. et al. Loss of the endothelial glycocalyx links albuminuria and vascular dysfunction. J. Am. Soc. Nephrol. 23, 1339-1350 (2012).

10. Sandoval, R. M., Wang, E. \& Molitoris, B. Finding the bottom and using it. Offsets and sensitivity in the detection of low intensity values in vivo with 2-photon microscopy. Intravital 2, 1-9 (2013).

11. Tanner, G. A. Glomerular sieving coefficient of serum albumin in the rat: a two-photon microscopy study. Am. J. Physiol. Renal Physiol. 296, F1258-F1265 (2009).

12. Centonze, V. E. \& White, J. G. Multiphoton excitation provides optical sections from deeper within scattering specimens than confocal imaging. Biophys. J. 75, 2015-2024 (1998).

13. Russo, L. M. et al. Impaired tubular uptake explains albuminuria in early diabetic nephropathy. J. Am. Soc. Nephrol. 20, 489-494 (2009).

14. Osicka, T. M. \& Comper, W. D. Protein degradation during renal passage in normal kidneys is inhibited in experimental albuminuria. Clin. Sci. 93, 65-72 (1997).

15. Russo, L. M. et al. Albuminuria associated with CD2AP knock out mice is primarily due to dysfunction of the degradation pathway processing of filtered albumin. FEBS Lett. 587, 3738-3741 (2013). 